\title{
Hybrid Molecular-Junction Mapping Technique for Simultaneous Measurements of Single-Molecule Electronic Conductance and Its Corresponding Binding Geometry in a Tunneling Junction
}

\section{Haijian Chen, Yunchuan Li, and Shuai Chang*}

The State Key Laboratory of Refractories and Metallurgy

The Institute of Advanced Materials and Nanotechnology

Wuhan University of Science and Technology

Wuhan, Hubei 430081, China

Table of Contents

S1. Home-built STM setup. $\mathrm{S}-2$

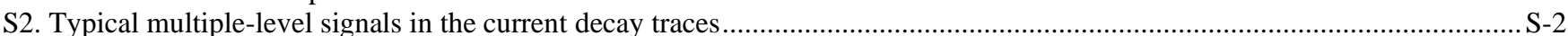

S3. Onset frequency of signals observed in the current decay traces with different tip retraction speed ..................................... S-2

S4. Analysis of a switching event .................................................................................................................................... S-3

S5. Typical traces for different molecules collected using break junction and molecular junction mapping techniques .................. S-4

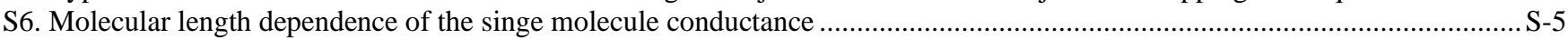

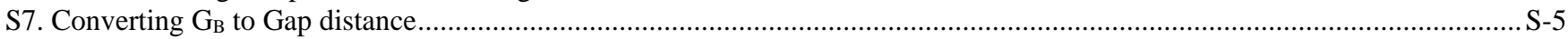

S8. Distribution of gap distances and durations of the molecular binding events ..................................................................... S-6

S9. Single molecule conductance of tilted BDA and HDA molecules in a tunneling junction .................................................. S-6

S10. Histogram of HDT molecular conductance taken by molecular junction mapping technique …........................................... S-7 


\section{S1. Home-built STM setup}

The experiments are carried out with a home-built single axis STM. The initial approach of the gold tip to the substrate is sequentially controlled by a stepper Picomotor (Newport 8742-4-KIT, Germany) and a 1-axis piezo actuator (S-303.0L PI, Germany, the spatial resolution is $0.03 \mathrm{~nm}$ ). During the conductance measurements, the tip movement is only controlled by the Piezo. To achieve a high spatial resolution, the voltage output to the piezo is provided by a NI PXIe-4463 card (24 bits) in the range of $-10 \mathrm{~V}$ to $10 \mathrm{~V}$ without amplification. The voltage output from the DAQ card has a resolution of $10 \mu \mathrm{V}$, resulting in a piezo displacement resolution of $0.03 \mathrm{~nm}$. A NI PXIe-4309 (18 bits at $2 \mathrm{MS} / \mathrm{s} / \mathrm{ch}$ ) is used to record the current, which is first amplified by a variable range currentvoltage converter (DLPCA-200, Femto, Germany). A custom LabVIEW program is coded to run the system, including hardware controlling and data acquisition.

\section{S2. Typical multiple-level signals in the current decay traces}

During the molecular junction mapping measurements, signals with multiple switching levels are occasionally observed, possibly reflecting the simultaneous binding activities of more than one molecule trapped in the gap. These signals are not included in the statistical analysis of single molecule conductance distribution.

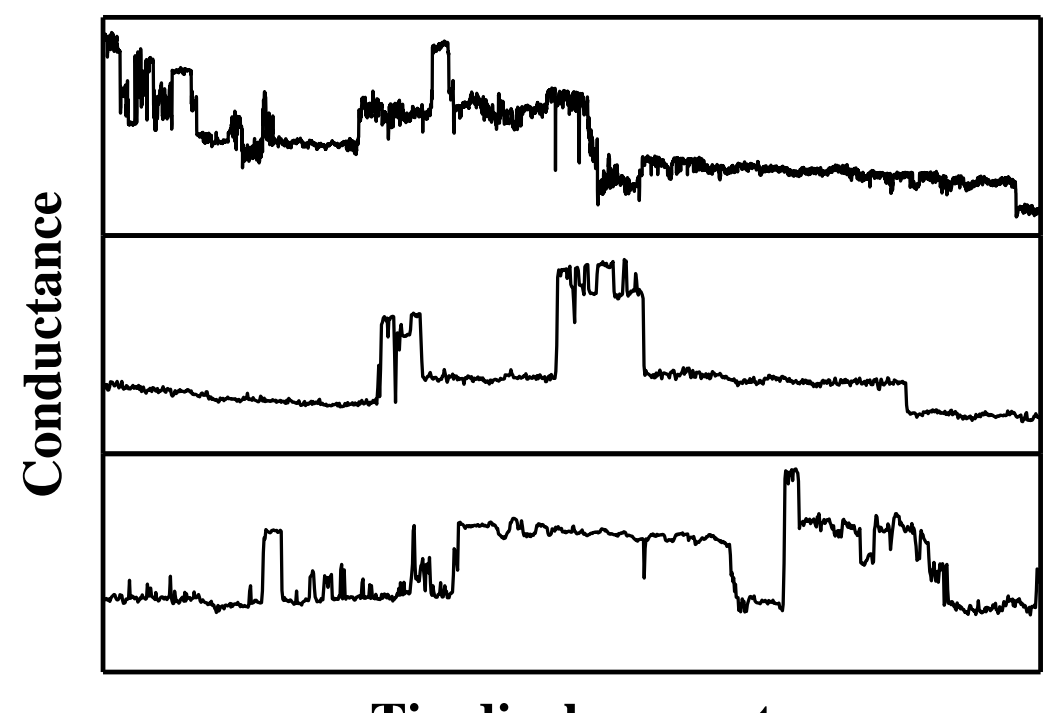

Tip displacement

Figure S1. Some typical multiple-level signals observed in typical current decay curves obtained in the molecular junction mapping measurements of HDA molecules.

\section{S3. Onset frequency of signals observed in the current decay traces with different tip retraction speed}

We repeated the molecular junction mapping measurements with different tip retraction speed at 0.2/0.5/1.0/2.0/5.0/10.0 nm/s, finding that current switching signals can be effectively generated only when the tip retraction speed is below $1 \mathrm{~nm} / \mathrm{s}$ for all three model molecules. 


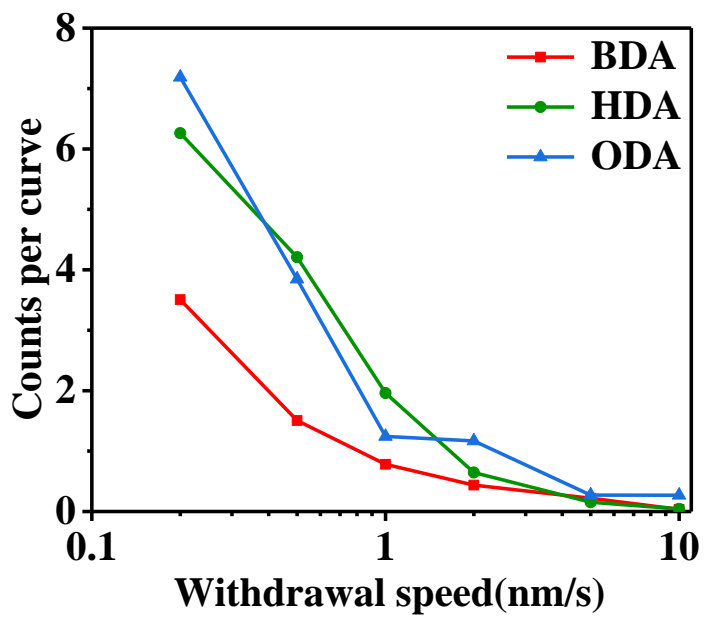

Figure S2. The average signal frequency observed in the molecular junction mapping measurements of BDA, HDA and ODA molecules collected at different tip retraction speed.

\section{S4. Analysis of a switching event}

Switching events are automatically detected and selected from each opening trace with a custom LabVIEW program. The peak detection algorithm is much as described in our earlier report. ${ }^{1}$ Figure S3a shows a typical raw switching event with its conductanceaxis plotted in logarithmic scale (duplicated from Figure 1d). In a tunneling model, conductance falls with distance as exp $(-\beta x)$. Since $\mathrm{x}$ is linearly related with time (the tip is pulled at a constant speed), the log-scale of the conductance will be linearly dependent by time. Therefore, we can linearly fit the background of the switching event plot in figure S3a based on the tunneling equation. The obtained $\beta$ from the fitted slope (blue line) is very close to the tunneling decay constant through solvent (see Figure S7), suggesting that the baseline conductance is the pure tunneling conductance. For extracting the parameters from each switching event, the background (blue line in figure S3a) is subtracted to obtain a spike plot with a flat baseline (c). It is then histogrammed and two distinct peaks can be obtained as shown in Figure d. The distance between the two peak centers is defined as the signal amplitude of $\mathrm{G}_{\mathrm{H}}$. The lifetime is determined by taking derivative of the raw switching signals, as displayed in (b), and the duration between the two apex points is defined as the lifetime. $\mathrm{G}_{\mathrm{B}}$ is determined by $\mathrm{y}$ value of the point in the fitted line with its $\mathrm{x}$ value corresponding to the middle point between the two apex points obtained in the derivative plot of Figure $b$.

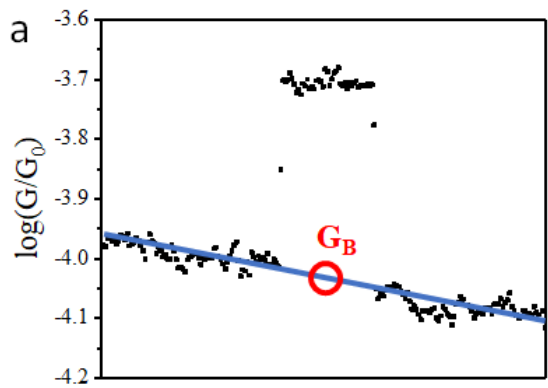

Time

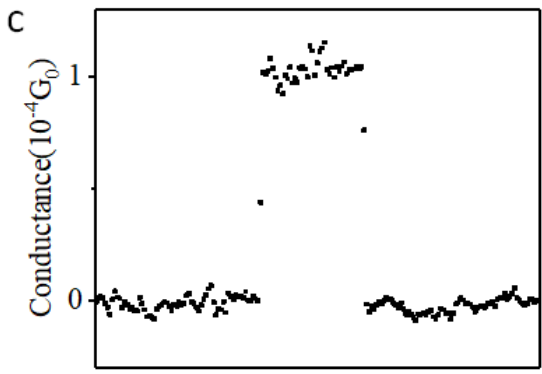

Time

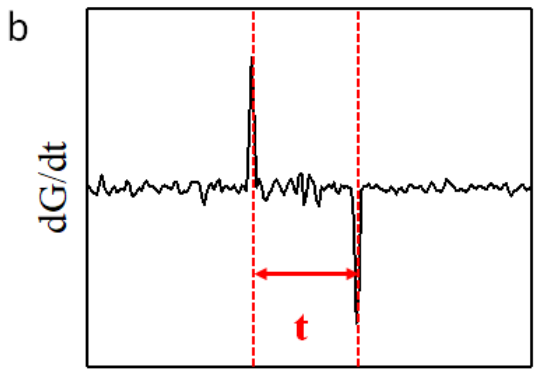

Time

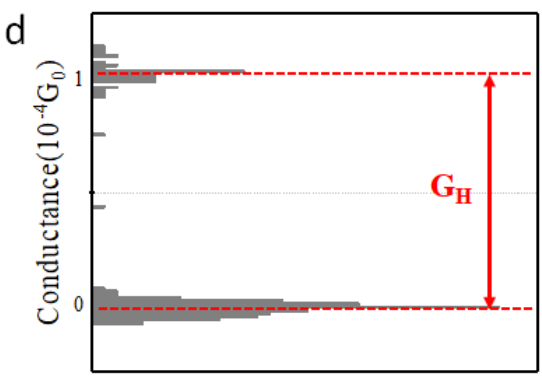

Counts

Figure S3. (a) A typical switching event with its background linearly fitted. (b) Derivative plot of the switching signal. (c) Switching signal plot with a flat baseline obtained by subtracting the fitted background. (d) The signal amplitude $\mathrm{G}_{\mathrm{H}}$ is defined as the distance between the two peaks in the amplitude histogram for each switching event 


\section{S5. Typical traces for different molecules collected using break junction and molecular junction mapping techniques}

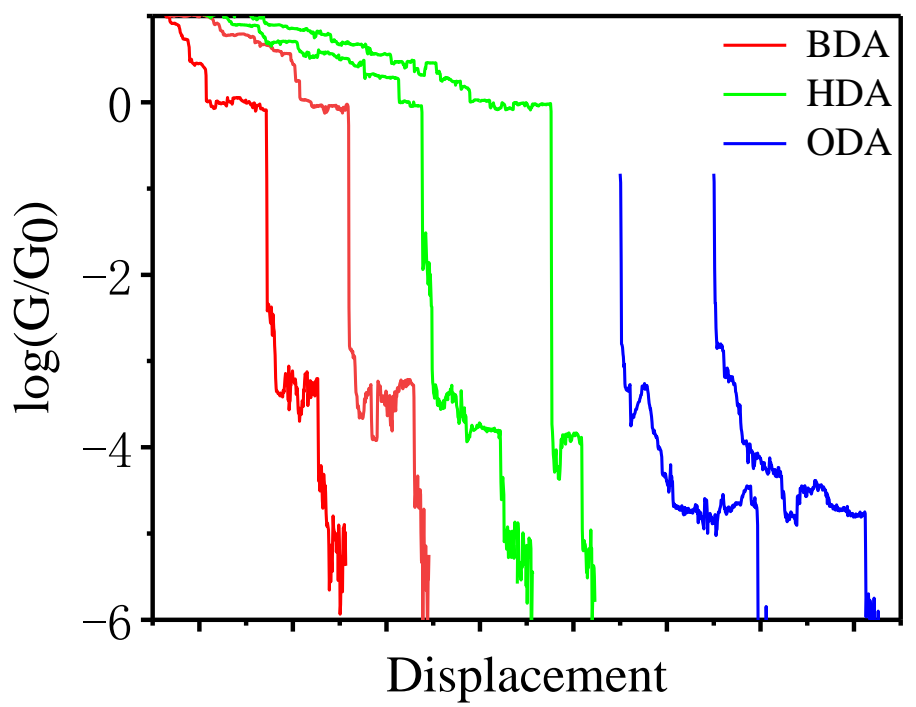

Figure S4. Typical break junction curves obtained with BDA, HDA and ODA molecules functionalized on the Au (111) surface. The tip retraction speed is $20 \mathrm{~nm} / \mathrm{s}$. The current preamplifier is set to $1 \mu \mathrm{A} / \mathrm{V}$ in the measurements of BDA and HDA molecules. It is changed to $100 \mathrm{nA} / \mathrm{V}$ in the measurements of ODA.

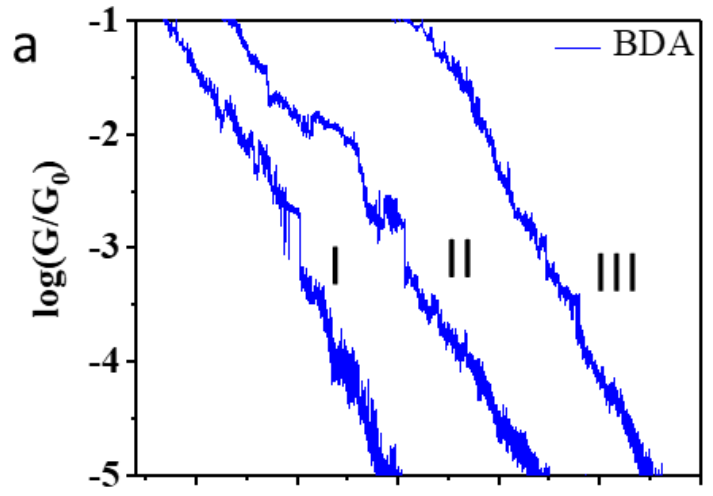

Tip displacem ent

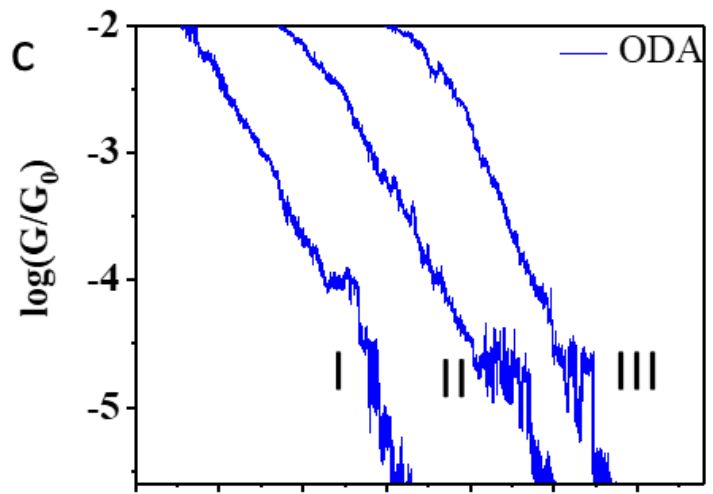

Tip displacement

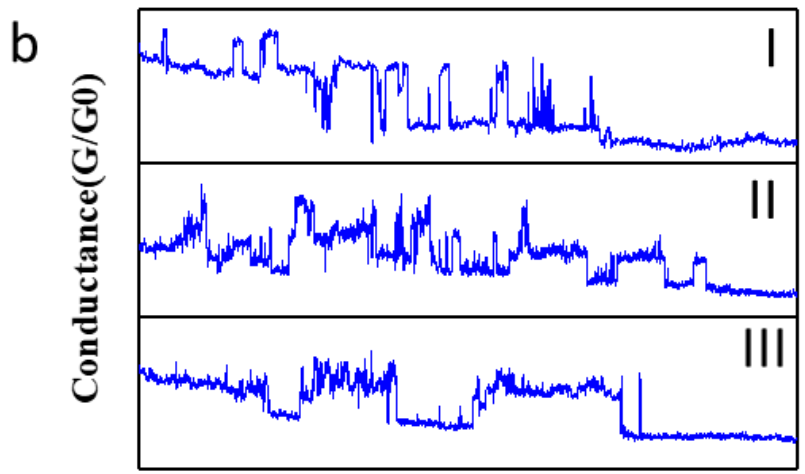

Tip displacement

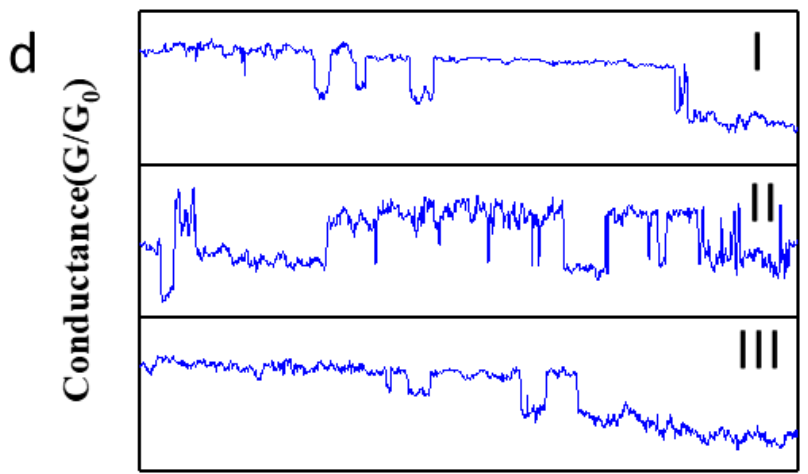

Tip displacement

Figure S5. Typical conductance decay curves obtained from measurements of BDA (a) and ODA (c) molecules and the zoomed in conductance fluctuations for BDA (b) and ODA (d) molecules. The tip retraction speed is $0.5 \mathrm{~nm} / \mathrm{s}$. 


\section{S6. Molecular length dependence of the singe molecule conductance}

The tunneling decay constant $\beta$ is obtained by fitting the scatter plots of the conductance versus the number of methylene groups. By extending the linear fits to intercept the zero methylene group axis, the intersection points are -3.19 and -2.56 in the unit of $\ln \left(\mathrm{G} / \mathrm{G}_{0}\right)$, which correspond to $314 \mathrm{k} \Omega$ and $167 \mathrm{k} \Omega$ for measurements with break junction and molecular junction mapping techniques.

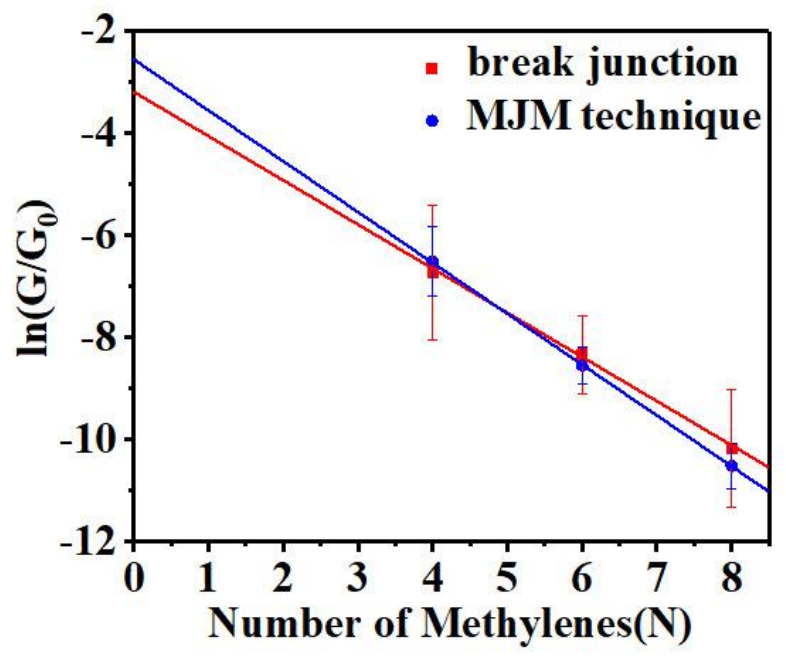

Figure S6. Linear fits to scatters plots of single molecule conductance versus the number of methylene groups.

\section{S7. Converting $G_{B}$ to Gap distance}

The gap distance s can be estimated based on Equation $\mathrm{S} 1^{2,3}$

$G=G_{0} * \exp (-\beta s)$

where $G$ is the baseline conductance, corresponding to $G_{B}$ in the main text, $G_{0}$ is the quantum of conductance (equals to $77.5 \mu S$ ) and $\beta$ is the tunneling decay constant through solvent. $\beta$ is obtained by fitting the curves of the current versus the distance change between electrodes. The current vs distance traces are taken in 1,2,4-tricholorobenzene solvent with target molecules. We select those scans in which current decays rapidly with no signs of molecular wire formations (i.e., current plateaus or spikes), and linearly fit the slope of $\ln \left(\mathrm{G} / \mathrm{G}_{0}\right)$ versus s to determine $\beta$, which is referenced from the previous reports. ${ }^{4-6}$ Examples of typical current decay traces and the fitting process are shown in Figure S7 $\mathrm{a}$ and $\mathrm{b}$. The obtained $\beta$ is $6.71 \pm 0.91 \mathrm{~nm}^{-1}$. We also measured pure TCB without target molecules and obtained $\beta \sim 7.18 \pm 0.79 \mathrm{~nm}^{-1}$. It is very close to the results in the previous reports. ${ }^{2,3}$

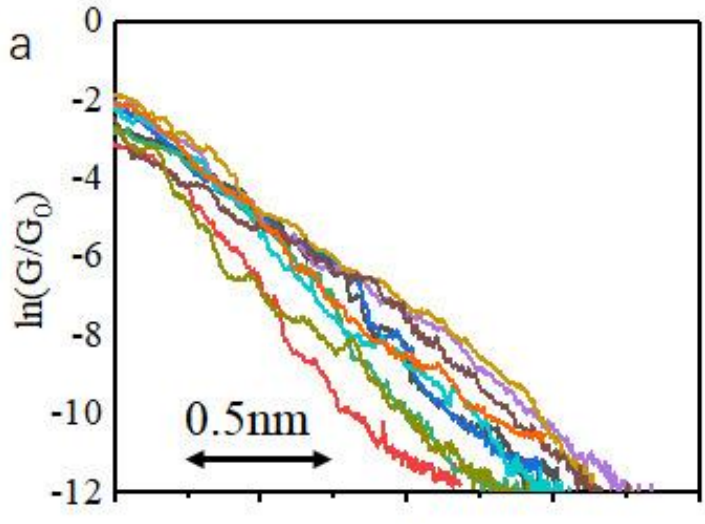

$\mathrm{S}$

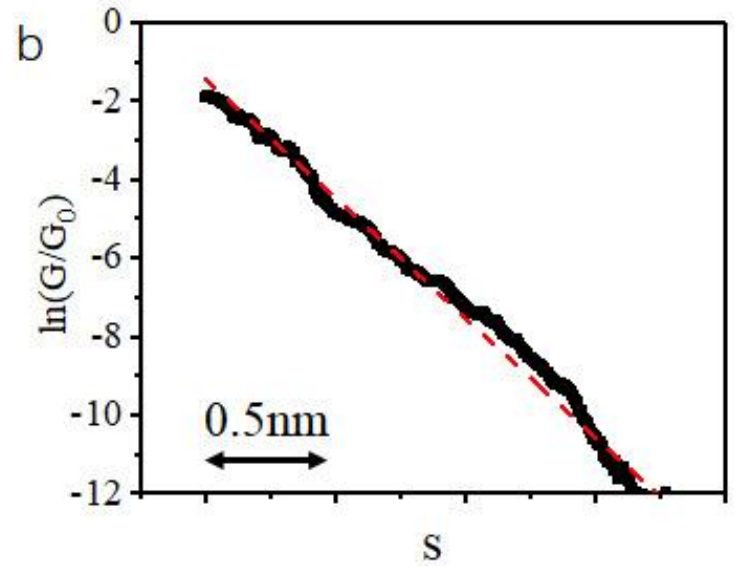

Figure S7. Semilogarithmic plot of the current versus the distance change between electrodes (a) and linear fitting (b) for the determination of tunnelling decay through solvent. 
S8. Distribution of gap distances and durations of the molecular binding events
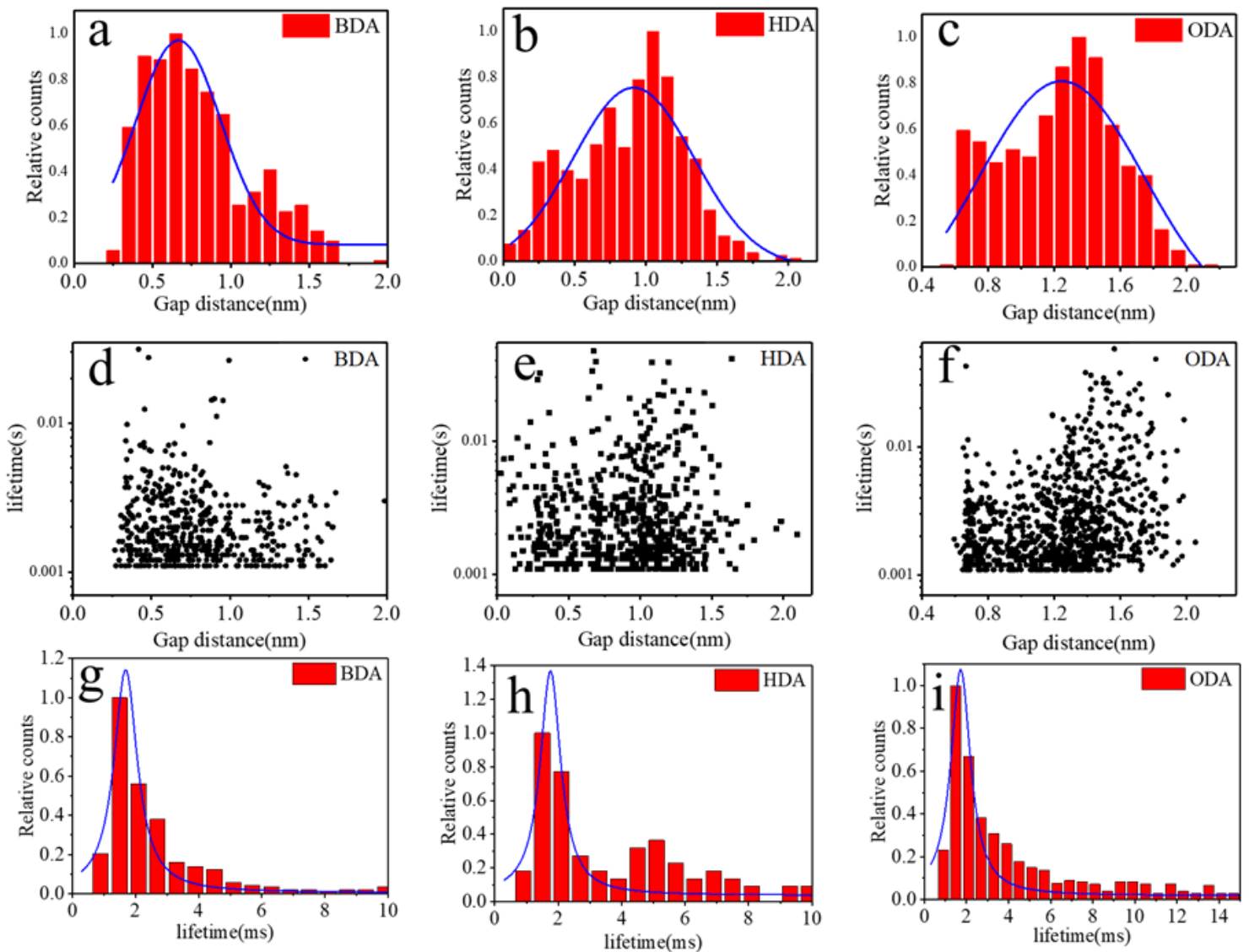

Figure S8. Distribution of gap distances for (a) BDA, (b) HDA and (c) ODA molecular binding events. (d-f) Event lifetime as a function of the gap distance and (g-i) the corresponding lifetime distributions for three molecules. Figure $\mathrm{h}$ is duplicated from Figure $1 \mathrm{f}$ in the manuscript for the ease of comparison.

S9. Single molecule conductance of tilted BDA and HDA molecules in a tunneling junction
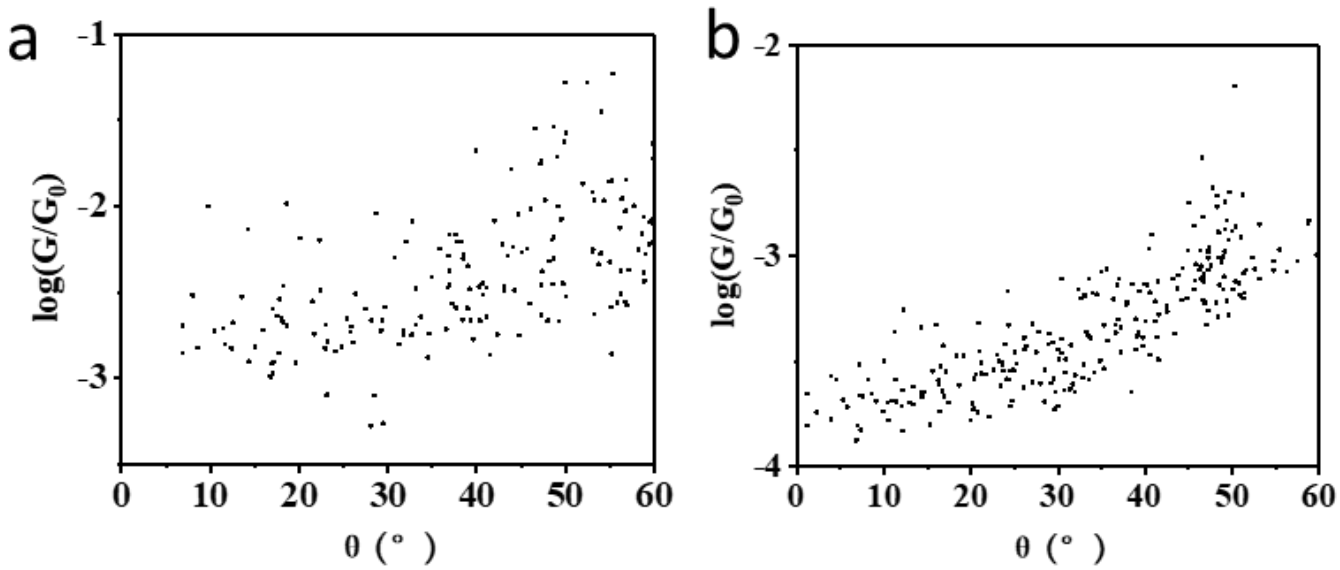

Figure S9. Molecular binding angle dependence of the single molecule conductance for BDA (a) and HDA (b) molecules in a tunnelling junction. $\theta$ is the angle between molecule and the Au surface normal. 


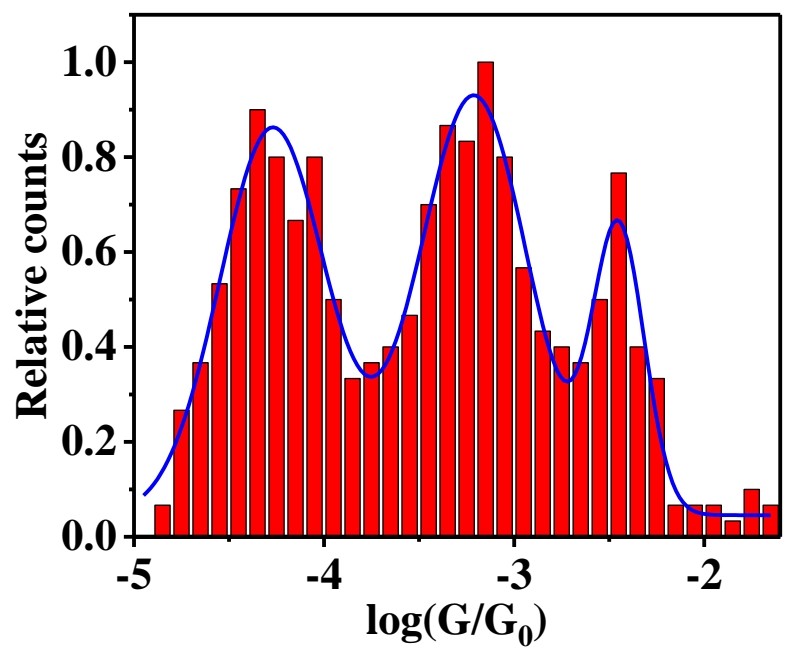

Figure S10. Conductance histogram of HDT molecules in a tunnelling junction. It is constructed from 100 typical traces without data selection.

Table S1: Calculated molecular length of three molecules by Chem3D.

\begin{tabular}{|l|l|l|l|}
\hline & BDA & HDA & ODA \\
\hline Molecular length $(\AA)$ & 6.274 & 8.804 & 11.378 \\
\hline
\end{tabular}

The length of three molecules was determined by Chem3D. Geometry optimization of three molecules was carried out using the Gaussian09 program employing the DFT incorporating Becke's three-parameter exchange augmented by Lee, Yang, and Parr's (B3LYP) correlation functional. Internally stored 6-31G*basis was used in these calculations.

\section{Reference}

1. Chang, S.; Huang, S.; He, J.; Liang, F.; Zhang, P.; Li, S.; Chen, X.; Sankey, O.; Lindsay, S., Electronic Signatures of all Four DNA Nucleosides in a Tunneling Gap. Nano Letters 2010, 10 (3), 1070-1075.

2. Xiao, B.; Liang, F.; Liu, S.; Im, J.; Li, Y.; Liu, J.; Zhang, B.; Zhou, J.; He, J.; Chang, S., Cucurbituril mediated single molecule detection and identification via recognition tunneling. Nanotechnology 2018, 29 (36), 365501.

3. He, J.; Lin, L.; Zhang, P.; Lindsay, S., Identification of DNA basepairing via tunnel-current decay. Nano Letters 2007, 7 (12), 3854-3858.

4. Haiss, W.; Wang, C.; Grace, I.; Batsanov, A. S.; Schiffrin, D. J.; Higgins, S. J.; Bryce, M. R.; Lambert, C. J.; Nichols, R. J., Precision control of single-molecule electrical junctions. Nat. Mater. 2006, 5 (12), 995-1002.

5. $\quad$ Martin, S.; Manrique, D. Z.; Garcia-Suarez, V. M.; Haiss, W.; Higgins, S. J.; Lambert, C. J.; Nichols, R. J., Adverse effects of asymmetric contacts on single molecule conductances of $\mathrm{HS}(\mathrm{CH} 2)(\mathrm{n}) \mathrm{COOH}$ in nanoelectrical junctions. Nanotechnology 2009, 20 (12), 9. 6. $\quad$ Haiss, W.; Martin, S.; Leary, E.; van Zalinge, H.; Higgins, S. J.; Bouffier, L.; Nichols, R. J., Impact of Junction Formation Method and Surface Roughness on Single Molecule Conductance. J. Phys. Chem. C 2009, 113 (14), 5823-5833. 